\title{
Simulative Analysis of 10 Gbps High Speed Free Space Optical Communication Link
}

\author{
Mehtab Singh \\ ECE Department \\ Satyam Institute of Engineering and Technology \\ Amritsar, India \\ mehtab91singh@gmail.com
}

\begin{abstract}
The increase in demand for high data transmission rates and channel capacity has led to the evolution of Free Space Optical (FSO) communication links. In this paper, performance analysis of a high-speed free space optical communication link has been observed. The performance of 10 Gbps FSO link has been evaluated on the basis of $Q$ Factor, SNR ratio, and total power of the received signal for a link distance ranging from $800 \mathrm{~m}$ to $2000 \mathrm{~m}$ at different power levels of data transmission and different levels of atmospheric attenuation. It has been seen from the results that the value of SNR of received signal lies in the range from $45 \mathrm{~dB}$ to $14 \mathrm{~dB}$ and $48 \mathrm{~dB}$ to $10 \mathrm{~dB}$ for the transmission power of $6 \mathrm{dBm}$ and attenuation of $25 \mathrm{~dB}$ respectively. Also, the value of the total power of received signal lies in the range of $-52 \mathrm{dBm}$ to $-73 \mathrm{dBm}$ and $-30 \mathrm{dBm}$ to -60 $\mathrm{dBm}$ for the transmission power of $6 \mathrm{dBm}$ and attenuation of $25 \mathrm{~dB}$ respectively.
\end{abstract}

Keywords: Free Space Optical Link; Q-Factor; SNR; received power; transmission power; attenuation

\section{Introduction}

In Free Space Optical (FSO) communication technique, light is used as a source to transmit information between two points in free space. This technology can be very useful where establishing a physical connection between transmitter and receiver is not feasible. The information transmission process is very similar to that in optical fiber communication in that information is transmitted using externally modulated laser light ( low data rate transmission over the short range is feasible using LED) [1]. Instead of transmitting light pulses through a glass fiber, the information signal is transferred in the form of a narrow beam of light through the atmosphere. The velocity of light in air is much faster than as compared to that in the glass. Hence, the free space optical communication link can easily be classified as optical communication at the velocity of light. The stability of the information transmission system and the quality of the received signal is highly dependent on the atmospheric conditions such as heat, haze, dust, rain, and fog. FSO communication links are considered for military applications due to their large number of inherent advantages as systems are rated with over I Km of transmission range in multiple lasers operating simultaneously to mitigate range related issues. The performance of data transmission system can be characterized by Q Factor, SNR, and total power of received signal [2]. Free Space Optical communication also called as Optical Wireless Communication system is a cost effective and highly attractive solution for information transmission at high data rates [3]. It has also presented an important solution for bottleneck connectivity problems and as an alternative to conventional techniques for data transmission such as $\mathrm{RF} /$ microwave communication links [4]. The effect on the performance of FSO communication link by snow and rain is quite low, but the performance is adversely affected by atmospheric conditions such as turbulence and 
dense fog. The value of attenuation coefficient ranges from $0.4 \mathrm{~dB} / \mathrm{Km}$ in the case of clear weather condition to $300 \mathrm{~dB} / \mathrm{Km}$ in the case of dense fog weather condition [5]. The quality of information transmission in an optical wireless communication system is mainly affected by scattering, absorption and scintillation. [6], [7]. These atmospheric effects can reduce the total power of received signal. The main reason for absorption is carbon dioxide and water vapors present in the air in the communication link path. The presence of water vapor and carbon dioxide is a function of altitude and humidity present in the air [8]. These effects can reduce the power density of transmitted signal in an FSO link and can reduce the availability of the system. However, in order to achieve high performance under the influence of such effects, appropriate transmission power and spatial diversity techniques (multiple light beams within an FSO link) can be used to maintain required link availability $[9,10]$.

The transmittance $\mathrm{T}$ of the laser radiation which has propagated over a link distance $\mathrm{L}$ is given by Bear's Law [11]

$\mathrm{T}=\exp \left(-\alpha_{e}(\lambda) \mathrm{L}[\mathrm{Km}]\right)$

Where $\alpha_{e}$ represents the extinction coefficient which indicates the level of extinction of the medium. Scattering results from the collision of light with the scatter. When the size of the scattering particle is comparable to that of the size of wavelength of optical signal, it is called as Mie Scattering. When the size of scattering particle is much smaller as compared to that of wavelength, it is called as Rayleigh scattering $[12,15]$. The scattering coefficient denoted by $\beta_{n}$ in clear weather condition depends on wavelength and is given by following equation [15]

$$
\beta_{n}=\frac{3.91}{V}\left(\frac{\lambda}{550 \mathrm{~nm}}\right)^{-Q}
$$

Where $\mathrm{V}$ denotes the visibility in $\mathrm{Km}$ and $\lambda$ denotes the wavelength of the optical signal in $\mathrm{nm}$. Q denotes the quality factor which is dependent on the size of scatter particles [15]. The value of $Q$ is 1.6 for visibility $(\mathrm{V}>60 \mathrm{Km}), 1.34$ for average visibility $(5<\mathrm{V}<60 \mathrm{Km}), 0.587 \mathrm{~V}^{1 / 3}$ for low visibility condition $(\mathrm{V}<5 \mathrm{Km})$.

When the values of molecular absorption coefficient, aerosol absorption coefficient, and Rayleigh coefficient are low, the extinction coefficient is given by [17]

$$
\alpha=\beta_{n}=\frac{3.91}{V}\left(\frac{\lambda}{550 \mathrm{~nm}}\right)^{-Q}
$$
[14]

The corresponding value of atmospheric attenuation is given by following equation

$$
\tau(R)=\frac{P(R)}{P(Q)}=\exp \left(-\beta_{n} R\right)
$$

In this paper, simulative investigation of a 10 Gbps Free Space Optical (FSO) communication link has been presented. Rest of the paper is organized as follows-: In Section 2, Simulation Setup is explained and results are discussed in Section 3. The overall conclusion from this simulative investigation is given in Section 4.

\section{Simulation Setup}

In this paper, the performance of an FSO communication link has been evaluated by using OPTISYSTEM simulation software which is a very powerful designing tool that allows modeling and simulating almost all types of optical communication links in the wide spectrum of optical links ranging from LAN to ultra-long haul. It can reduce the amount of time required and reduces the cost needed in designing of optical links and components. Several system parameters can be varied to get desirable system performance. The FSO communication link model is illustrated in Fig. 1. The proposed design of FSO link in this paper consists of an optical transmitter section which is further 
divided into three subsections. The first sub-section consists of a Pseudo-Random Bit Sequence Generator (PRBS). This subsystem represents the data this is supposed to be transmitted in the form of binary signals consisting of a stream of 1's and 0 's of a known pattern. The second subsystem consists of an Non Return to Zero (NRZ) pulse generator. This subsystem performs the function of encoding the binary data from PRBS generator to electrical pulses of NRZ format. The third subsystem consists of a Mach-Zender modulator which modulates the electrical signal from NRZ pulse generator with a continuous wave Laser [7].

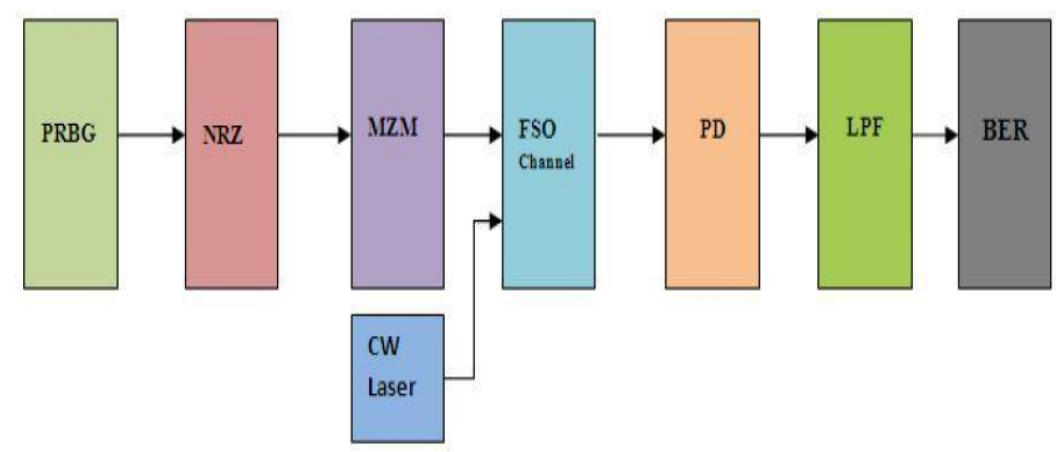

Figure 1. Free Space Optical Communication Link

The free space in between the transmitter and the receiver is considered as the Free Space Optical Communication channel which is the medium of propagation for the light transmitted. In this paper, OPTISYSTEM simulation software is used to design an FSO communication link between the transmitter and the receiver with antenna aperture diameter of $30 \mathrm{~cm}$ on each side. Also, the beam divergence angle is set to the value of 3 mrad for simulation purpose. The receiver section of the FSO link consists of an APD photo detector which converts the received optical signal to electrical signal. The APD used must be capable of meeting the bandwidth requirements of the system. The signal from APD is directed towards the LPF which removes any high-frequency noise present in the received signal. The LPF Bessel filter used in this simulation has a cut-off frequency of $0.75 *$ bit rate of the signal. BER analyzer is used to analyze the performance of received signal in the form of Q Factor, total power received and SNR. Table 1 shows the various simulation parameters considered in this paper.

Table 1. Simulation Parameters

\begin{tabular}{|c|c|}
\hline Parameter & Description/ Value \\
\hline Transmitting Wavelength & $1550 \mathrm{~nm}$ \\
\hline Transmitting Optical Power & $4-8 \mathrm{dBm}$ \\
\hline Transmitter aperture diameter & $30 \mathrm{~cm}$ \\
\hline Link Distance & $2000 \mathrm{~m}$ \\
\hline APD multiplication factor & 1 \\
\hline Data rate & $10 \mathrm{Gbps}$ \\
\hline APD quantum efficiency & 0.8 \\
\hline Filter Type & Bessel Filter \\
\hline APD dark current & $1 \mu \mathrm{A}$ \\
\hline Divergence angle & $3 \mathrm{mrad}$ \\
\hline
\end{tabular}

\section{Results and Discussions}

In this paper, the performance of an FSO communication link is analyzed by varying the data transmission power levels and attenuation of free space communication channel 
and the link range between the transmitter and the receiver. The system performance is analyzed on the basis of Q Factor, SNR and total power of received signal.

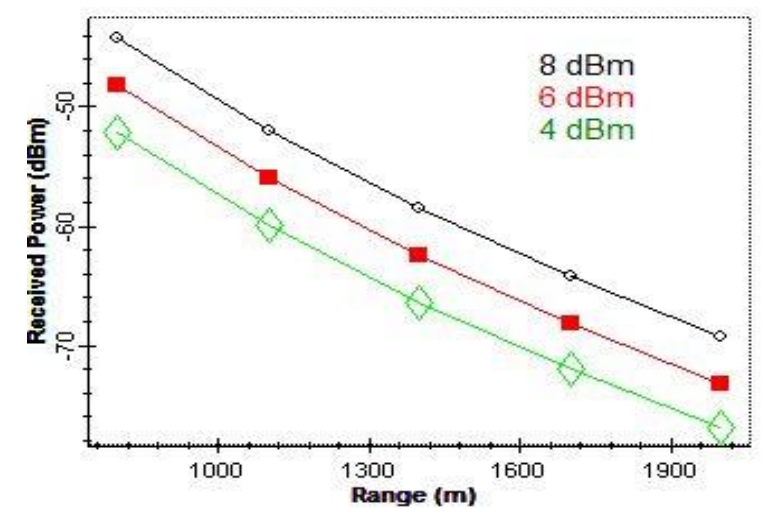

Figure 2(a). Received Power for Different Transmission Power Levels

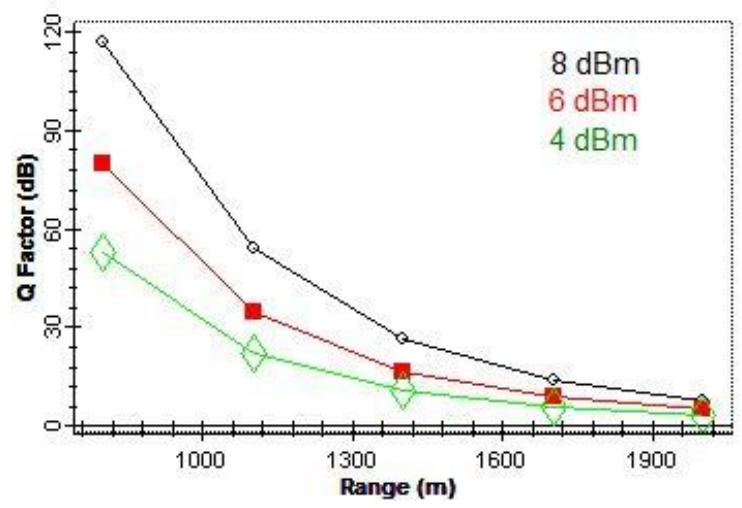

Figure 2(b). Q Factor for Different Transmission Power Levels

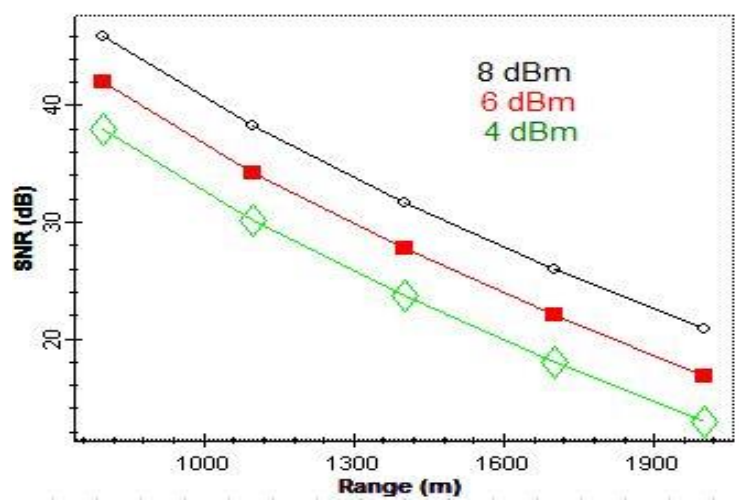

Figure 2(c). SNR for Different Transmission Power Levels

Figure 2(a), 2(b), and 2(c) indicate the graph between received power, Q Factor and SNR for varying link distance at different levels of transmission powers. It can be observed from the results that there is decrease in received power which lie in the range [$43,-47,-52]$ to $[-68,-72,-80] \mathrm{dBm}$ for link distance of $2 \mathrm{Km}$ in case of $8 \mathrm{dBm}, 6 \mathrm{dBm}$, and $4 \mathrm{dBm}$ respectively and Q Factor values changes from $[120,87,56]$ to $[11,9,7] \mathrm{dB}$ for link distance of $2 \mathrm{Km}$ in case of $8 \mathrm{dBm}, 6 \mathrm{dBm}$, and $4 \mathrm{dBm}$ respectively and SNR lie 
in the range $[48,42,39]$ to $[20,18,12] \mathrm{dB}$ for link distance of $2 \mathrm{Km}$ in case of $8 \mathrm{dBm}, 6$ $\mathrm{dBm}$, and $4 \mathrm{dBm}$ respectively.

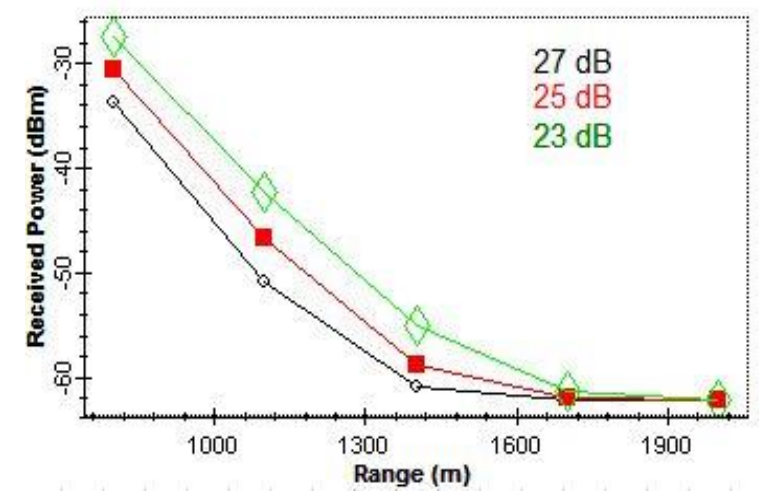

Figure 3(a). Received Power for Different Attenuation Levels

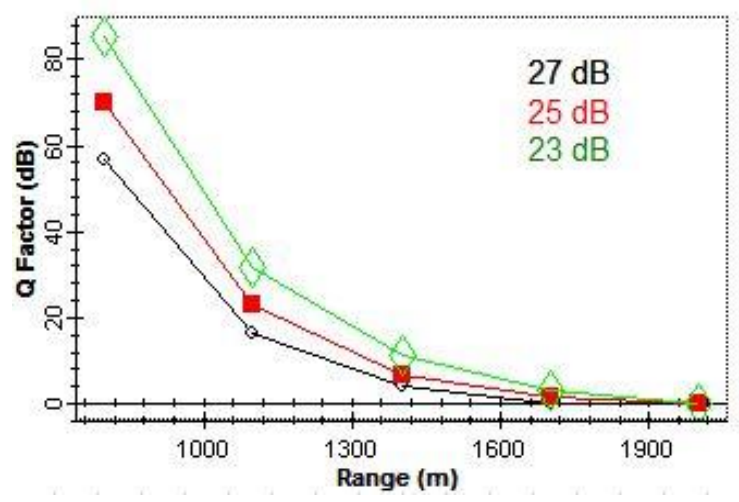

Figure 3(b). Q Factor for Different Attenuation Levels

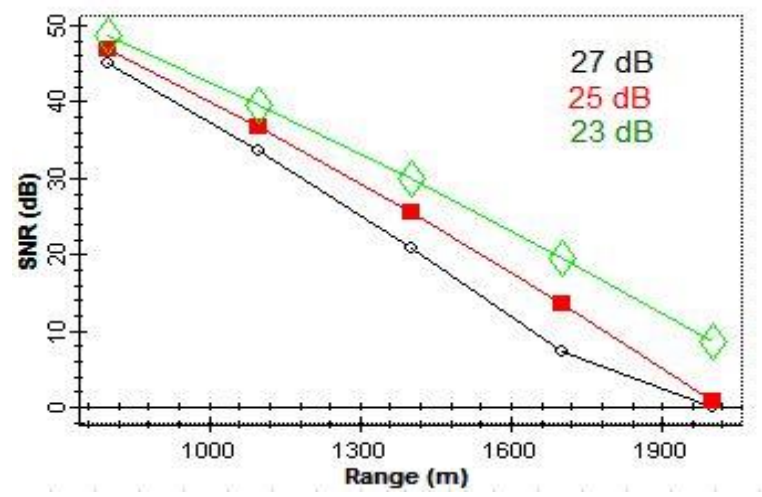

Figure 3(c). SNR for Different Attenuation Levels

Figure 3(a), 3(b), and 3(c) indicate the graph between received power, Q Factor and SNR for varying link distance at different levels of attenuation. It can be observed from the results that there is decrease in received power which lie in the range $[-28,-30,-38]$ to $[-52,-55,-60] \mathrm{dBm}$ for link distance of $1.5 \mathrm{Km}$ in case of $23 \mathrm{~dB} / \mathrm{Km}, 25 \mathrm{~dB} / \mathrm{Km}$, and 27 $\mathrm{dB} / \mathrm{Km}$ attenuation respectively and $\mathrm{Q}$ Factor values changes from $[84,70,60]$ to $[18,15$, 10] $\mathrm{dB}$ for link distance of $1.5 \mathrm{Km}$ in case of $23 \mathrm{~dB} / \mathrm{Km}, 25 \mathrm{~dB} / \mathrm{Km}$, and $27 \mathrm{~dB} / \mathrm{Km}$ attenuation respectively and SNR lies in the range $[48,46,34]$ to $[22,19,10] \mathrm{dB}$ for link 
distance of $1.5 \mathrm{Km}$ in case of $23 \mathrm{~dB} / \mathrm{Km}, 25 \mathrm{~dB} / \mathrm{Km}$, and $27 \mathrm{~dB} / \mathrm{Km}$ attenuation respectively.

\section{Conclusion}

In this paper, the impact of an information signal transmission power level and attenuation levels on the performance of Free Space Optical Communication system has been analyzed and discussed. Performance has been analyzed on the basis of Q Factor, SNR, and total power of received signal. From the results, it can be observed that $\mathrm{Q}$ Factor lies in the range 120 to $10 \mathrm{~dB}$ and total received power lies in the range -43 to -68 $\mathrm{dBm}$ and SNR lies in the range 48 to $20 \mathrm{~dB}$ for the transmission power of $8 \mathrm{dBm}$ and link distance of $2 \mathrm{Km}$. Also, Q Factor lie in the range 84 to $18 \mathrm{~dB}$ and total received power lies in the range -28 to $-52 \mathrm{dBm}$ and SNR lies in the range 48 to $22 \mathrm{~dB}$ for attenuation of 23 $\mathrm{dB} / \mathrm{Km}$. It can be concluded that there is a significant improvement in Q Factor, total received power, and SNR for transmission power level of $8 \mathrm{dBm}$ in FSO link. Also, any further increase in transmission power levels is not permitted as it may cause harm to human skin and eyes. It can also be concluded that the quality of the received signal improves as the attenuation levels decrease.

\section{References}

[1]. H. Henniger and O. Wilfert, "An Introducttion to Free Space Optical Communications.", Radioengineering, vol. 19, (2010).

[2]. B. Barua, "Comparison the Performance of FreeSpace Optical Communication with OOK and BPSK Modulation under Atmospheric Turbulence", International Journal of Engineering Science and Technology (IJEST), vol. 3, (2011)

[3]. W.O. Popoola, "BPSK Subcarrier Intensity Modulated Free-Space Optical Communications in Atmospheric Turbulence", Journal of Lightwave Technology, vol. 27, (2009).

[4]. Nadeem, E. Leitgeb, and M.S. Awan, "Optical Wavelengths Comparison for Different Weather Conditions", IEEE, (2009).

[5]. B. Epple, "Simplified Channel Model for Simulation of Free-Space Optical Communications", vol. 2, (2010).

[6]. K. Kiaseleh, "Performance of Coherent DPSK Free Space Optical Communication System in Distributed Turbulence", IEEE Transactions Communication, vol. 54, (2006).

[7]. "Info-Communications Development Authority (IDA) of Singapore", A Trial-Based Study of Free-Space Optics Systems in Singapore, (2002).

[8]. I. I. Kim, B. McArthur and E. Korevaar, "Comparison of Laser Beam Propagation at 785nm and 1550nm in Fog and Haze for Optical Wireless Communications", SPIE Proceedings, vol. 4214, pp. 26-37.

[9]. H. Nistazakis, T.A. Tsiftsis and G.S. Tombras, "Performance Analysis of Free Space Optical Communication Systems over Atmospheric Turbulence Channels", IET Communication, vol. 3, no. (8), pp. 1402-1408.

[10].H. Manor and S. Arnon, "Performance of an Optical Wireless Communication System as a Function of Wavelength, Applied Optics, vol. 42, no. 21, pp. 4285-4293.

[11].A.S. Mohd Supa'at, A.B. Mohammad, Y.T. Tong and S.M. Idrus, "Unguided Optical Communication: Design a", (1999).

[12].S. M. Mndewa, D. Huang and X. Yuan, "A Survey of Atmospheric Turbulence on Laser Propagation. Asian Journal of Information Technology", vol. 7, no. 7, pp. 307-312.

[13].B. S. Naimullah, M. Othman, A. K. Rahman, S. I. Sulaiman,, S. Ishak, S. Hitam and S. A. Aljunid, "Comparison of Wavelength Propagation for Free Space Optical Communications. International Conference of Electronic Design", (2008), pp. 1-5.

[14].I. I Kim, R. Stieger, J. A. Koontz, C. Moursund, M. Barclay, P. Adhikari, J. Schuster and E. Korevaaret, "Wireless Optical Transmission of Fast Ethernet, FDDI, ATM and ESCON Protocol Data Using the TerraLink Laser C", (1998).

[15].O. Bouchet, H. Sizun, C. Boisrobert, F. de Fornel and P.N. Favennec, "FreeSpace Optics:Propagation and Communication", publisher Hermes Science in France, 2004nd Evaluation in Malaysia Atmosphere", (2004), pp. 269-271. 